Background Adolescent is a time of heightened violence and the frequency of engaging in violent behaviour is greater for adolescents than for all other age groups. A better understanding of factors related to assault among adolescents is important to reduce the incident. This study described and characterised the sociodemographic factors, circumstances and characteristic of assault in emergency department.

Methods A descriptive study was carried on 140 patients at ER, Yangon General Hospital during one year. Adolescent assault victims with musculoskeletal injuries were collected involving demographic details, circumstances of injury, characteristics of injury and initial treatment parameter.

Results Among the 140 respondents, males (80\%) and females (20\%) reported been an assault. Males were more likely to have been in a physical fight. The majority of cases $(47.1 \%)$ were of low educational status (primary education). Casual workers and dependent were the most common group among the injured, occurred at public place during night time. Blunt object (81\%) were the most common weapons used. Head and neck were commonly affected in over half of cases (58\%) and soft tissue injuries (90\%) were the most frequent type. $71 \%$ of the patients were treated as out-patients.

Conclusion assaults are responsible for significant morbidity and may be an important opportunity for prevention. Socioeconomic status, contributing factors and injury characteristics identified to this study should help strategies for preventing adolescent assault.

\section{CHARACTERISTICS OF UNINTENTIONAL AND INTENTIONAL CHILD INJURIES FROM EMERGENCY AND OUTPATIENT ROOM IN CHINA, 2006-2014}

Wang Yuan, Ye Pengpeng, Duan Leilei. National Centre for Chronic and Noncommunicable Disease Control and Prevention, Chinese Centre for Disease Control and Prevention

\subsection{6/injuryprev-2016-042156.688}

Background Injury is the first death cause of children aged 0 to 17 in China. Prevention of unintentional and intentional injury was totally different.

Methods Data collected from National Injury Surveillance System that based on emergency and outpatient department of 126 sentinel hospitals around the whole country through 2006 to 2014 were analysed. Mechanism, place where injury occurred, severity and outcome of unintentional and intentional injuries among patients aged 0 to 17 were summarised.

Results Among the 895,243 patients aged 0-17, 94.22\% cases were unintentional injuries; $4.84 \%$ cases were intentional injuries (interpersonal violence cases: $4.50 \%$, self-injuries: $0.34 \%$ ).

The top injury mechanisms were falling (51.95\%), road traffic injury (14.36\%), blunt injury (9.59\%), animal injury $(9.11 \%)$ and sharp injury (6.88\%) among unintentional injuries; blunt injury $(63.73 \%)$, sharp injury (12.01\%), animal injury (7.94\%), falling $(4.35 \%)$, poisoning $(0.56 \%)$ among interpersonal violence cases; poisoning (51.96\%), sharp injury (21.29\%), falling $(10.60 \%)$, blunt injury $(5.66 \%)$, road traffic injury $(3.70 \%)$ among self-injuries.

$40.35 \%$ of unintentional injuries occurred in home, followed by road (19.53\%), school and public place (17.63\%), public housing (15.50\%); for interpersonal violence cases, $47.64 \%$ occurred in school and public place, followed by public housing (19.60\%), home (10.69) and road (8.95\%); for self-injuries, $65.08 \%$ occurred in home, followed by school and public place (12.79\%), public housing (10.04\%) and road (3.63\%).

Among unintentional injuries, $81.62 \%$ were mitigated injuries, $17.21 \%$ were moderate injuries and $1.16 \%$ was severe injuries. The proportion of mitigated, moderate and severe injuries among interpersonal violence cases and self-injuries were $79.53 \%$, $18.88 \%, 1.59 \%$ and $51.55 \%, 38.36 \%, 10.08 \%$ respectively.

$86.42 \%$ of unintentional injuries were discharged, $12.96 \%$ were hospitalised and $0.09 \%$ died; the proportion of being discharged, being hospitalised and dying among interpersonal violence cases and self-injuries were $80.16 \%, 18.96 \%, 0.08 \%$ and 54.32\%, 43.98\% , 1.08\% respectively.

Conclusions It's necessary to have a good understanding of the characteristics of child injuries for child injury prevention.

\section{BRITISH PARENTS' SELF-REPORTED ROAD SAFETY BELIEFS, PRACTICES AND RULES}

'Karen Pfeffer, ${ }^{2}$ Zahra Tabibi. ${ }^{1}$ University of Lincoln, UK; ${ }^{2}$ Ferdowsi University of Mashhad, Iran

\subsection{6/injuryprev-2016-042156.689}

Background Children are at high risk for pedestrian deaths and injury (World Health Organisation, 2009). Parents play an important role in teaching children pedestrian skills in context. The aims of this research were to investigate parents' self-reported beliefs, practices and road safety rules when crossing streets with their children.

Methods A questionnaire was administered to parents of 234 children aged $3-10$ years $($ mean $=6.23$ years, $S D=1.83)$. Participants were parents of children who were attending a university children's event in a small British city. The majority of respondents were mothers. Questions were about pedestrian experiences, safety rules and risks, also who they think is best suited to teach children about road safety.

Results The majority (61.5\%) normally walked to school on a daily basis. The age parents thought it was appropriate for boys and girls to walk to school alone was significantly lower than the appropriate age to walk to school with peers. Parents ranked themselves as the most suited to teach children about road safety in comparison to professionals such as teachers and road safety education officers. Only 3 parents said that they did not have any specific road safety rules and the majority of parents reported that they hold their child's hand when crossing the road. The road safety rules that parents reported were similar across child age groups.

Conclusions Our sample of parents reported that they walk with their children frequently. Parents' self-reported rules and behaviour did not differ with child age. The younger age at which British parents considered it appropriate for children to walk to school with peers compared to alone needs further investigation. In this respect, further research should consider comparisons of road risk and 'stranger danger' (abduction risk). Also, further research should focus on reasons that parents considered road safety professionals less suited to teach children about road safety than parents. 